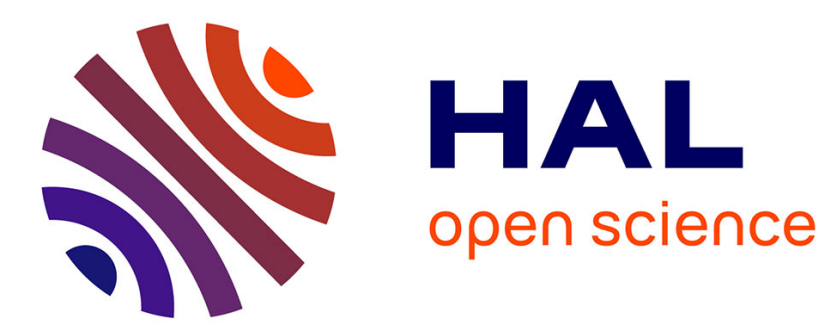

\title{
Interaction with Artificial Companions: Presentation of an Exploratory Study
}

Matthieu Courgeon, Charlotte Hoareau, Dominique Duhaut

\section{To cite this version:}

Matthieu Courgeon, Charlotte Hoareau, Dominique Duhaut. Interaction with Artificial Companions: Presentation of an Exploratory Study. Eight International Conference on Social Robotics (ICSR 2016), Nov 2016, Kansas City, MO, United States. pp.611-620, 10.1007/978-3-319-47437-3_60 . hal01413375

\section{HAL Id: hal-01413375 \\ https://hal.science/hal-01413375}

Submitted on 19 Sep 2017

HAL is a multi-disciplinary open access archive for the deposit and dissemination of scientific research documents, whether they are published or not. The documents may come from teaching and research institutions in France or abroad, or from public or private research centers.
L'archive ouverte pluridisciplinaire HAL, est destinée au dépôt et à la diffusion de documents scientifiques de niveau recherche, publiés ou non, émanant des établissements d'enseignement et de recherche français ou étrangers, des laboratoires publics ou privés. 


\title{
Interaction with Artificial Companions: Presentation of an Exploratory Study
}

\author{
Matthieu Courgeon ${ }^{1}$, Charlotte Hoareau ${ }^{2}$, Dominique Duhaut ${ }^{2}$ \\ ${ }^{1}$ Lab-STICC - ENIB, Brest France \\ ${ }^{2}$ Lab-STICC - University of South Brittany
}

\begin{abstract}
The MoCA project aims to design and study children-companion relationship through virtual agents, personal robots and communicating devices. In this article, we present an exploratory study of the free 30min long interactions between children and a set of artificial, robot-like and virtual companions. We present a preliminary overview of the results obtained pending further analyses.
\end{abstract}

Keywords: Artificial companions; robotics; interaction; serious games

\section{Introduction}

Technologies of artificial companions have evolved remarkably over the last few years. These developments are related to an of intensive research activity in the scientific communities focused on serious games [1,2], affective computing [2], and personal robotics $[3,4]$.

This research shows the need to consider the role which the virtual companions can play - or should or should not play - in our daily lives [5]. The exploratory study of Dautenhahn et al. [3] shows that, in the studied population, $40 \%$ of the respondents are not opposed to the idea of having an artificial companion, provided its role is limited to that of a personal assistant. On the other hand, few of them accept the idea that the companion can be a friend or an intimate companion.

The study of human-robot interactions from a social perspective is still relatively new compared to conventional or industrial service robotics, which requires only a minimal interaction between human and robot $[6,7]$. However, there are numerous potential fields of application, i.e.: assistance for the elderly [8,9] and with children $[4,10$, 11]. There is no shortage of research undertaken along these lines [12]. Nevertheless, the studies carried out are often restricted to interactions of short duration on a single task, which constrains the user, who is the subject of the experiment, thereby orienting his or her perception of the potential of robot-like devices.

The experiment implemented here within the framework of the MoCA project aims at offering a larger range of possible interactions, not only concerning the nature of the devices, but also the diversity of the activities available. 


\section{The MoCA Project}

MoCA (in French: Mon petit monde de Compagnons Artificiels - My little world of artificial companions) is a fundamental research project which studies artificial companions (virtual characters and personal robots) for users in everyday life situations.

At the outset of the MoCA project, the type scenario was defined as follows: "artificial companions to accompany a child alone at home in different problem situations: comfort, safety, assistance with activities, games, etc.” The exploratory study presented here is the result of an integration of the research tasks undertaken by the four IT laboratories belonging to the consortium.

\subsection{Possible roles for artificial companions}

We define the various roles that the companions should be able to fulfil. These roles were divided into five categories. The study was designed so these various roles could be performed as well as possible, although, some of them were left out of account.

Help with extra-curricular activities/Coaching: Encouraging physical activity, Motivation, Valorization, Sharing non-formal knowledge, Reading of current events, follow-up of information

Homework support: Appraisal, Motivation, Valorization, Adaptation, Teaching with the child, Feedback to parents and teachers

Comfort, company: Ability to listen, give advice, Showing empathy

Child monitoring, safety: Detection of danger, Raising alarm, Contact adults, Recommendations and advice

Entertainment: Proposing games, Forming a pattern of interaction with 1 child, 1 child with n companions, $\mathrm{m}$ children with $\mathrm{n}$ companions

\section{Presentation of the experimentation}

Based on this general definition, we chose to conduct the experiment in a "natural environment" in which the child would actually be placed in the situation presented above. It proved too complicated to equip the home environment of each subject, so to create a comparable situation, we rented a conventional apartment which was then arranged and equipped with the artificial companions and materials necessary for the study (see plan on Fig. 1).

Among the five types of problem situations described above, we chose not to consider point number 4 (Child monitoring, safety) in this experimentation. Since the child was not in a usual situation, we did not wish to place it in a situation of stress or at risk.

Several activities were proposed both with virtual agents and robots, based on technological capacities and limitation of each items. The activities installed in the apartment, which correspond to the four other roles, are as follows: 


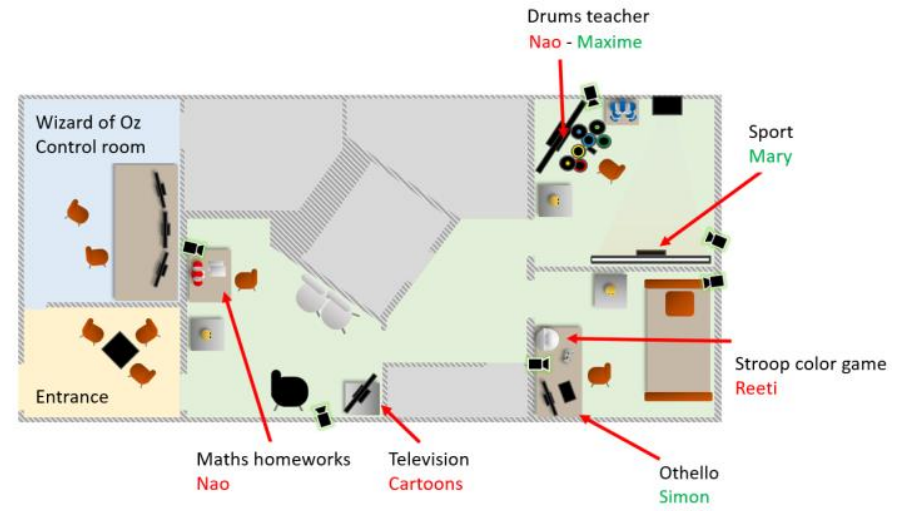

Fig. 1. Floor plan of the apartment

Role 1: Help and Coaching with extra-curricular activities

- Sports game/exercise with a projected life-size virtual character.

- Introduction to playing drums (percussion) with a virtual character and a Nao robot to study the synergy between virtual reality and robotics.

Role 2: Homework support

- Maths exercise (geometry) on paper with a Nao robot, teaching assistance, motivation and reminder of course material.

Role 3: Company

- Support in the choice of activities by a dedicated robot companion (Keepon), listening and general interaction, and overall coherence of the interaction. While the Keepon is not mobile, we have three identical keepons, one per room, allowing for a continuous interaction. We briefly considered allowing the child to carry Keepon with $\mathrm{him} / \mathrm{her}$, but it would have been prone to technical issues.

Role 5: Entertainment and games

- Colour memory games with a Reeti robot.

- Board games with a virtual agent on screen.

Further details on some of these activities can be found on related publications, about the technologies and activities developed during the MoCA project $[13,14,15,16]$.

\subsection{Methodology}

The study presented here is of an exploratory nature. It involves analysing the interactions between the participants and the system in the most environmentally friendly context possible. During these free interactions with the system, it is possible to collect chronometric, behavioural and subjective data. Analysis of these data allows us to identify the types of interactions that are accepted and appreciated, and conversely, those interactions and companion behaviours that are considered to be problematic, unacceptable, or simply inconvenient. 
Unlike traditional experimental approaches, establishing a set of research hypotheses and then identifying all the independent and dependent variables that will manipulated to test these hypothesis, we adopt an exploratory approach. In practice, rather than defining several groups of subjects facing distinct controlled setup, we make each participant evolve in the system at will and we analyze his/her behavior retrospectively, without printing a priori assumptions on the system. According to the classification of experimental approaches by Trudel et al. [17], this approach aims to "circumscribe an object of research, identify new research directions, choose theoretical avenues or identify an appropriate method to study the object".

In our case, the object of the study is large and too complex to be handled simply. The interaction effects between the parameters are too numerous and involve a great number of assumptions to control. The resulting combinatorial experimental conditions would require a very high number of subjects that we simply cannot have. The exploratory approach therefore seems appropriate to identify research directions to dig in more detail later.

\subsection{Recruitment of the participants}

The study was aimed at children in their final classes of primary education ( 9 or 10 $y-o$ ). To recruit the participants, we approached nearby primary schools and, with the agreement of the head teachers of the targeted schools, we circulated a call for participation. The call was designed to prompt the curiosity of the children and parents, but divulged almost nothing about the study itself. The participants spontaneously contacted us for an appointment to take part in the study. The experimental sessions involved a total of 20 participants aged between 9 and 11 years, coming from three different primary schools (14 males, 6 females).

The sessions were filmed in their entirety by six cameras (two axes per room), with a microphone on the child, and a microphone in the vision/sound control room to record the reactions of the relative.

\section{Procedure of a standard experimental session}

Phase 1 - We welcomed the child and relative in a reception area (at bottom on the left in Fig. 1) and they signed a mutual assent document. The child is then equipped with a microphone. We explained to the child that he will be alone and will interact with artificial companions and let him/her enter the apartment while the relative goes into the technical control room with the experimenters.

Phase 2. Presenting the whole set of possible activities to the child, as well as the companions with which it will be able to interact.

When the child enters, a Keepon robot is activated and gives a welcome, proposing to start by visiting the apartment. Each activity is presented by the companion with which it is specifically associated. For example, in the first room, maths is presented by the red Nao on the desk. 
At the end of the visit, the child is asked to fetch a teddy bear, which is in the "drums/sport" room, and to bring it back into the first room. The objective of this action is to neutralize the short-term memory effect when the first activity is chosen, and also to avoid that the last activity presented is always the one wanted by the child. In addition, this request also enables us to test the capacity of the child to obey a request given by a robot in the first minutes of their interaction.

Phase 3 - Once the rooms have been visited in their entirety (and at the end of each activity), the Keepon asks the child which activity it wishes to carry out. If the child chooses an activity, it is then triggered. In the (frequent) case where the child does not choose an activity, a suggestion/acceptance/refusal procedure is triggered.

At the start of an activity, the companions comment on the preceding activity (or the fact that the child chooses to start with them). This mechanism is implemented to reinforce the illusion of continuity and the existence of a "world of companions".

After thirty minutes, the Keepon invites the child to leave.

Phase 4 - Semi-structured interviews

Once the experiment is finished, the child returns to the reception area where it is questioned by one of the experimenters, initially according to a precise questionnaire, then in semi-structured interviews. During this time, the other experimenter takes the relative into the apartment to visit what was seen on the control screens, and also carries out a semi-structured interview.

\section{Questionnaires and Interviews}

\subsection{Debriefing with the child}

The questionnaire is composed of a set of 13 questions relating to the usefulness, usability and acceptability of the companions, as well as the perception of their expressivity. To the best of our knowledge, no standardized questionnaire is available to evaluate such a complex system. We thus designed our own set questions.

The semi-structured interviews were carried out by revisiting the apartment with the child, in order to reactivate his or her experience in situ.

The topics addressed by the interviews are composed of open questions divided into four categories:

1 - Going back in a general way to what happened

2 - Focusing on the most striking aspects

3 - General feeling of the child towards the companions and activities

4 - Perceptions of emotions expressed by the companions

Parts of the topics addressed here are deliberately duplicated in the questionnaire given in the first part of the debriefing. These overlaps make it possible to corroborate the results.

\subsection{Visit with the relative}

The visit with the relative consisted solely of a semi-structured interview. For each activity, the relative had to answer the following questions and give a justification if he 
or she so wished: a) Is this activity acceptable with a companion? b) Would you let your child do this activity alone? Once the visit was finished, some more general questions were put to the relative: a) Broadly, do you find that such a device is useful? b) Would you use it if it were free? Would you buy it? c) Would you hand over part of the supervision of your children to an automated system? d) What are your feelings about the overall behaviour of your child in front of these activities?

\section{$5 \quad$ Preliminary Results}

The results of the study are being analysed. The video recordings are in the course of annotation. In this article, we present only a preliminary analysis of the results from the children's questionnaires.

The results presented here relate to 16 subjects aged between 9 and 10 years. Four participants had to be excluded from the analysis, either because of technical errors of the system, or because of their age: the 11-year old subject, in the first form of secondary school, showed a behaviour that contrasted too greatly with the 9- and 10-year-old children. The results include a group of 16 children (13 boys and 3 girls) with an average age of 9.3 years (range: 9 to 10 years).

For the purpose of the analysis, the results from the questionnaire are placed in four categories: (1) usefulness, (2) usability, (3) acceptability and (4) credibility and expression of emotions. The questions relating to the first three categories are built on a rating scale qualifying the degree of agreement in terms of three types of response, which are coded numerically as follows: 1 negative, 2 neutral and 3 positive. The averages and standard deviations given here are based on this coding.

\subsection{Usefulness of the device}

Four questions of the questionnaire aim to assess the children's perception of the degree of usefulness of artificial companions as guides for carrying out daily activities.

An analysis of the results of the question: "Are [companions] good for keeping you company when you are alone at home?" shows that 12 of the 16 respondents gave a positive response ("Completely in agreement"), as against 1 who were undecided ("Perhaps") and 3 with a negative response ("Not at all"; $\mathrm{m}=2.56 ; \sigma=0.81$ ).

Two questions relate to the usefulness of artificial companions for entertainment activities. Results for question no. 4 : "Are companions better for having fun than video games or television?" show that 10 of the 16 respondents consider that taking part in activities accompanied by artificial companions is "Much better" than playing video games or watching television, while 5 find the activities equally good ("Similar") and 1 find them "less good" $(\mathrm{m}=2.56 ; \sigma=0.62)$.

Results for question no. 5: "Do you think an artificial companion is better for having fun than playing with your friends?" show that taking part in activities with artificial companions, compared to activities with friends, is never considered as "much better", but is regarded as equivalent ("Similar") by 12 of the 16 children and "less good" by 4 of them $(\mathrm{m}=1.75 ; \sigma=0.45)$. 
The last question about the degree of usefulness of artificial companions is concerned with the activity of homework support (question: "Is it better to have a companion while doing your homework than when alone?"). The results show that none of the respondents prefers to do homework alone ("Less good"), while 3 children do not feel any difference between doing homework alone or with an artificial companion ("Similar") and 13 find an advantage in being accompanied by an artificial companion ("Much better", $\mathrm{m}=2.81 ; \sigma=0.40)$.

An analysis of the answers to questions on artificial companions for accompanying daily activities shows that children mainly perceive the usefulness of such devices in relation to educational activities and as a substitute for multimedia activities.

\subsection{Usability of the device}

The usability of artificial companions, and by extension, the activities which they propose, is evaluated in the questionnaire through two questions requiring the children to judge the ability of other pupils in their class to use these devices.

An analysis of the responses given to the question "Do you think that the other children in your class could easily use these activities?" shows that 14 of the 16 respondents consider that other children would manage to use the device "Rather easily" and 2 "Very easily" $(\mathrm{m}=2.13 ; \sigma=0.34)$.

The second question is "[Did other children in your class] need your help to use the device?" The answers are divided between "Not at all" for 7 of the 16 respondents and "Perhaps" for 8 children. No respondents answered "Yes, definitely" to this question $(\mathrm{m}=2.44 ; \sigma=0.51)$.

None of the children gave a negative response to either of these questions, indicating an absence of any difficulty in using the set up implemented in this study.

\subsection{Acceptability of the device}

The concept of acceptability relates to the decision whether or not to use the device when it is in our possession. This concept is assessed in the questionnaire by posing two questions.

The first question is: "If the [artificial companions] belonged to you, would you be pleased to show them to your friends?" The majority of the children 13) are willing to show their artificial companions "Only to best friends" and 3 to "All friends". None of the children consider that they would show them to "Anybody" $(\mathrm{m}=2.19 ; \sigma=0.44)$.

The second question is: "Do you consider it enjoyable or annoying to have all these systems in your home?" Among all the children, 9 find it enjoyable to have artificial companions in their home and 7 do not find it "Inconvenient". None of children find the presence of artificial companions "Inconvenient" $(\mathrm{m}=2,56 ; \sigma=0.51)$.

Generally, the respondents are not opposed to the presence of artificial companions in their home or among their circle of friends. 


\subsection{Credibility and expression of emotions}

The four questions relate to the perception of various emotions expressed by the artificial companions. The children were asked whether they perceived the artificial companions as angry, happy or sad ("Did you see at least one of the companions being angry/happy/sad?"). An analysis of the responses shows variations in the perception of emotions between different children. For example, 4 of the children detected anger in at least one companion $(\mathrm{m}=0.25 ; \sigma=0.45)$ and 3 did not describe any artificial companion as being happy $(\mathrm{m}=0.81 ; \sigma=0.40)$.

In a general way, the artificial companions manage to transmit emotional states, indicating the non-unanimous perception of anger, happiness and sadness. The children also differentiate the behaviours of the artificial companions; for example, the Nao robot is considered "stupid" because of its humorous remarks during the activity of initiation to drumming.

\section{Conclusion}

In this article, we present a complex study that is rich in interactions, integrated within the scope of the MoCA project. In this study, 20 children aged from 9 to 11 years were free to interact with a world of companions made up of 6 robots and 3 virtual agents, involved in a series of 5 game and teaching activities.

Although analysis of the data is still in progress, we present here some preliminary results based on the responses given by the children to a subjective questionnaire. Although these results are insufficient when considered on their own, they nevertheless provide some valuable insights to support the behavioural data based on video recordings that will be analysed in the second phase of the study.

Our preliminary results suggest that:

a. Artificial companions are perceived as especially useful in relation to educational activities (homework) and as a replacement for multimedia activities (video games and television).

b. The usability of the presented devices appears to be good. The children consider that it is easy for them, as well as others in the same age group, to take part in the activities. We had anticipated remarks about the fact that certain activities were difficult to perform (for example: drums for those who do not play a musical instrument, maths for children in difficulty, or games for children who do not have a video game console in their home). However, no remarks of this type were made.

c. In a general way, the children included in this study are not against the presence of artificial companions in their home or among their circle of friends. They were satisfied with the interaction and seem to have appreciated the experiment. Compared to the results of Dautenhahn et al. [3], it would appear that children are more inclined to accept this type of technology than adults. Moreover, all the children indicated that, to varying degrees, they were in favour, "of showing their companions to their friends". This finding suggests that having this kind of device would be a matter of pride, and not an embarrassment. Thus, artificial companions may be regarded as representing social values that can be shared and compared. These results should clearly be qualified 
and put into perspective using the answers given by the parents during semi-structured interviews.

d. As regards the expression of emotional states, we note that children perceive the emotions shown by virtual characters. The nature of the perceived emotions is variable, because their expression depends on the choices made by the child during the experimental session and his or hers varying rate of success in the interaction. Nevertheless, it would appear that children interacting with artificial companions perceive a form of social presence.

Before leaving, one of participants spontaneously took the time to thank several of the companions: "Thank you very much, that was very good... all the activities. Goodbye". This type of behaviour clearly shows that the companions are perceived as social entities which children consider as having their "own lives". Besides, most of the children indicated that they perceived what we call the "world of companions"; more precisely, they feel there is an extremely strong social bond between the companions, which makes the "collective" set up richer than a simple sum of activities with each of them taken independently.

These preliminary results are therefore very encouraging. The data collected should lead to a detailed behavioural analysis allowing us to shed light on many examples of child-companion interactions. Owing to the large freedom of choice and variability of the activities proposed in this study, it is impossible to carry out a systematic processing of the data. Nevertheless, in our opinion, this type of approach yields additional results that are broader in scope and more instructive when compared with classical laboratory studies, which are more restricted and more tightly controlled. In the future, we intend to examine more closely the data collected during this preliminary study, so the further results of this research can be made available.

\section{$7 \quad$ Acknowledgements}

This work was carried out in the framework of a project aimed at designing a world of artificial companions, which is financed by the project ANR-2012-CORD-019-02 "Mon Petit Monde de Compagnons Artificiels: MoCA". http://www.moca.imag.fr

\section{$8 \quad$ References}

1. Castellano, G., Leite, I., Pereira, A., Martinho, C., Paiva, A., \& McOwan, P. W. (2010). Affect recognition for interactive companions: challenges and design in real world scenarios. Journal on Multimodal User Interfaces, 3(1-2), 89-98.

2. Pereira, A., Leite, I., Mascarenhas, S., Martinho, C., \& Paiva, A. (2010). Using empathy to improve human-robot relationships. In Human-Robot Personal Relationships (pp. 130-138). Springer Berlin Heidelberg.

3. Dautenhahn, K., Woods, S., Kaouri, C., Walters, M. L., Koay, K. L., \& Werry, I. (2005, August). What is a robot companion-friend, assistant or butler?. In Intelligent Robots and Systems, 2005.(IROS 2005). 2005 IEEE/RSJ International Conference on (pp. 1192-1197). IEEE. 
4. Nalin, M., Baroni, I., Sanna, A., \& Pozzi, C. (2012, June). Robotic companion for diabetic children: emotional and educational support to diabetic children, through an interactive robot. In Proceedings of the 11th International Conference on Interaction Design and Children (pp. 260-263). ACM.

5. C. Clavel, C. Faur, J. -C. Martin, S. Pesty and D. Duhaut, (2013) Artificial Companions with Personality and Social Role - Expectations from Users and Impact on the Design of Groups of Companions, IEEE SSCI 2013 Singapore

6. D.Duhaut., (2015) Keywords and dimensions of artificial companions, 7th IEEE International Conference on Cybernetics and Intelligent Systems (CIS) and the 7th IEEE International Conference on Robotics, Automation and Mechatronics (RAM), July 2015, Angkor Wat, Cambodia.

7. D. Wilkes, R. Alford, R. Pack, R. Rogers, R. Peters, and K. Kawamura, "Toward socially intelligent service robots," Applied Artificial Intelligence Journal, vol. 12, pp. 729-766, 1997.

8. Sidner, C. L., Rich, C., Shayganfar, M., Bickmore, T. W., Ring, L., \& Zhang, Z. (2015). A Robotic Companion for Social Support of Isolated Older Adults. In HRI (Extended Abstracts) (p. 289).

9. Wang, R., Zhang, H., \& Leung, C. (2015). Follow Me: A Personal Robotic Companion System for the Elderly. International Journal of Information Technology, vol 21(1).

10. Nalin, M., Bergamini, L., Giusti, A., Baroni, I., \& Sanna, A. (2011, March). Children's perception of a robotic companion in a mildly constrained setting. In IEEE/ACM Human-Robot Interaction 2011 Conference (Robots with Children Workshop) Proceedings.

11. Iacono, I., Lehmann, H., Marti, P., Robins, B., \& Dautenhahn, K. (2011, August). Robots as social mediators for children with Autism-A preliminary analysis comparing two different robotic platforms. In IEEE International Conference on Development and Learning (ICDL), 2011 (Vol. 2, pp. 1-6). IEEE.

12. W.Johal, C.Adam, H.Fiorino, S.Pesty, C.Jost and D.Duhaut. (2014) Acceptability of a companion robot for children in daily life situations IEEE 5th International Conference on Cognitive Infocommunications, November 2014 Vietra

13. Courgeon, M., Martin, J. C., \& Jacquemin, C. (2008). Marc: a multimodal affective and reactive character. In Proceedings of the 1st Workshop on AFFective Interaction in Natural Environments.

14. Courgeon, M., Céline, C., \& Martin, J. C. (2014, May). Modeling facial signs of appraisal during interaction: impact on users' perception and behavior. In Proceedings of the 2014 international conference on Autonomous agents and multi-agent systems (pp. 765-772). International Foundation for Autonomous Agents and Multiagent Systems.

15. Faur, C., Clavel, C., Pesty, S., \& Martin, J. C. (2013, September). PERSEED: a self-based model of personality for virtual agents inspired by socio-cognitive theories. In Affective Computing and Intelligent Interaction (ACII), 2013 Humaine Association Conference on (pp. 467-472). IEEE.

16. M. Courgeon, D. Duhaut (2015) Artificial Companions as Personal Coach for Children: The Interactive Drums Teacher, in: 12th Internationnal Conference on Advances in Computer entertainment Technologies - ACE 2015, 16-19 nov. 2015, Malaysie

17. L. Trudel, C. Simard, N. Vonarx (2007) La recherche qualitative est-elle nécessairement exploratoire ?, Recherches Qualitative, Les Questions de l'Heure- Hors Série - numéro 5 - pp. 38-45, 